\title{
Evolution of the pore structure during the early stages of the alkali-activation reaction: an in situ small-angle neutron scattering investigation
}

Claire E. White ${ }^{1 *}$, Daniel P. Olds ${ }^{2,3}$, Monika Hartl ${ }^{2,4}$, Rex P. Hjelm² and Katharine Page ${ }^{2,3}$

\author{
${ }^{1}$ Department of Civil \& Environmental Engineering and Andlinger Center for Energy and the \\ Environment, Princeton University, Princeton, USA \\ ${ }^{2}$ Lujan Neutron Scattering Center, Los Alamos National Laboratory, Los Alamos, USA \\ ${ }^{3}$ Present address: Spallation Neutron Source, Oak Ridge National Laboratory, Oak Ridge, USA \\ ${ }^{4}$ Present address: European Spallation Source, Lund, Sweden
}

\section{Supporting Information}




\section{Unified Fits of the Alkali-Activation Reaction}

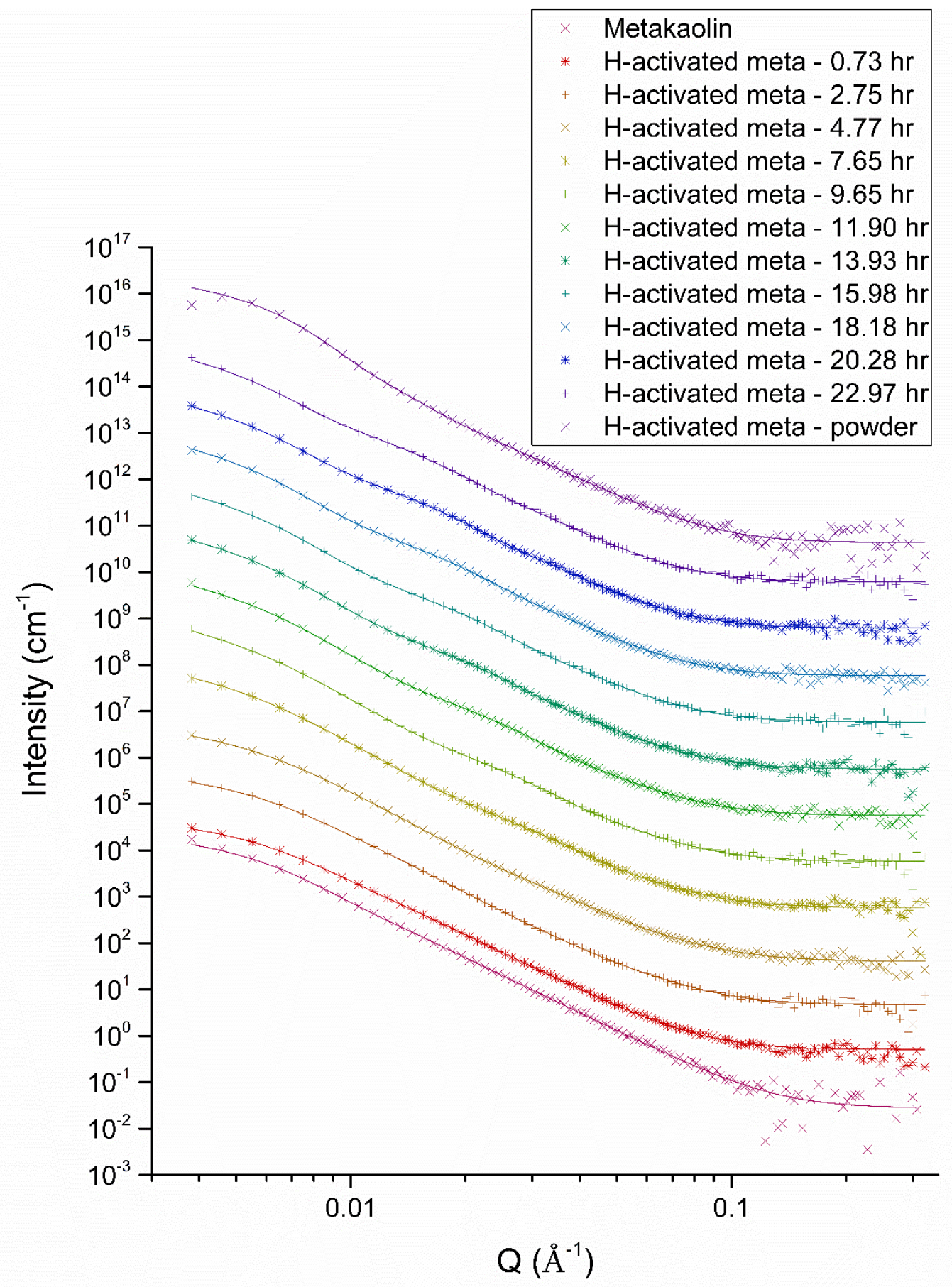

Figure S1. Small-angle neutron scattering pattern of dry metakaolin powder and patterns of the $\mathrm{H}$ activated metakaolin paste during the alkali-activation reaction. The lines represent the unified fit results. Error bars are excluded for clarity, but are comparable to those given in Figure 9 in the main article. Data are offset by multiples of ten. 


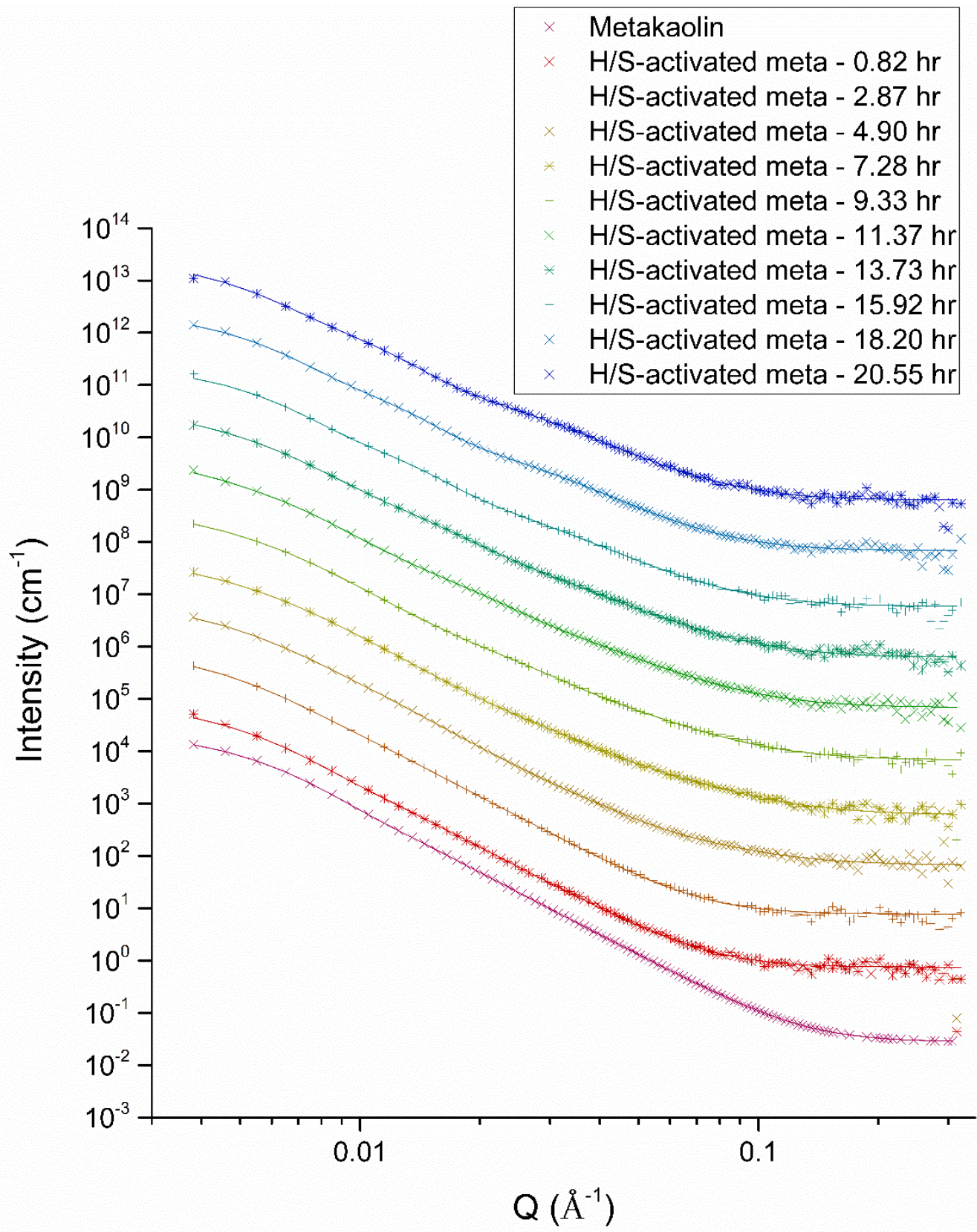

Figure S2. Small-angle neutron scattering pattern of dry metakaolin powder and patterns of the $\mathrm{H} / \mathrm{S}$-activated metakaolin paste during the alkali-activation reaction. The lines represent the unified fit results. Error bars are excluded for clarity, but are comparable to those given in Figure 9 in the main article. Data are offset by multiples of ten. 


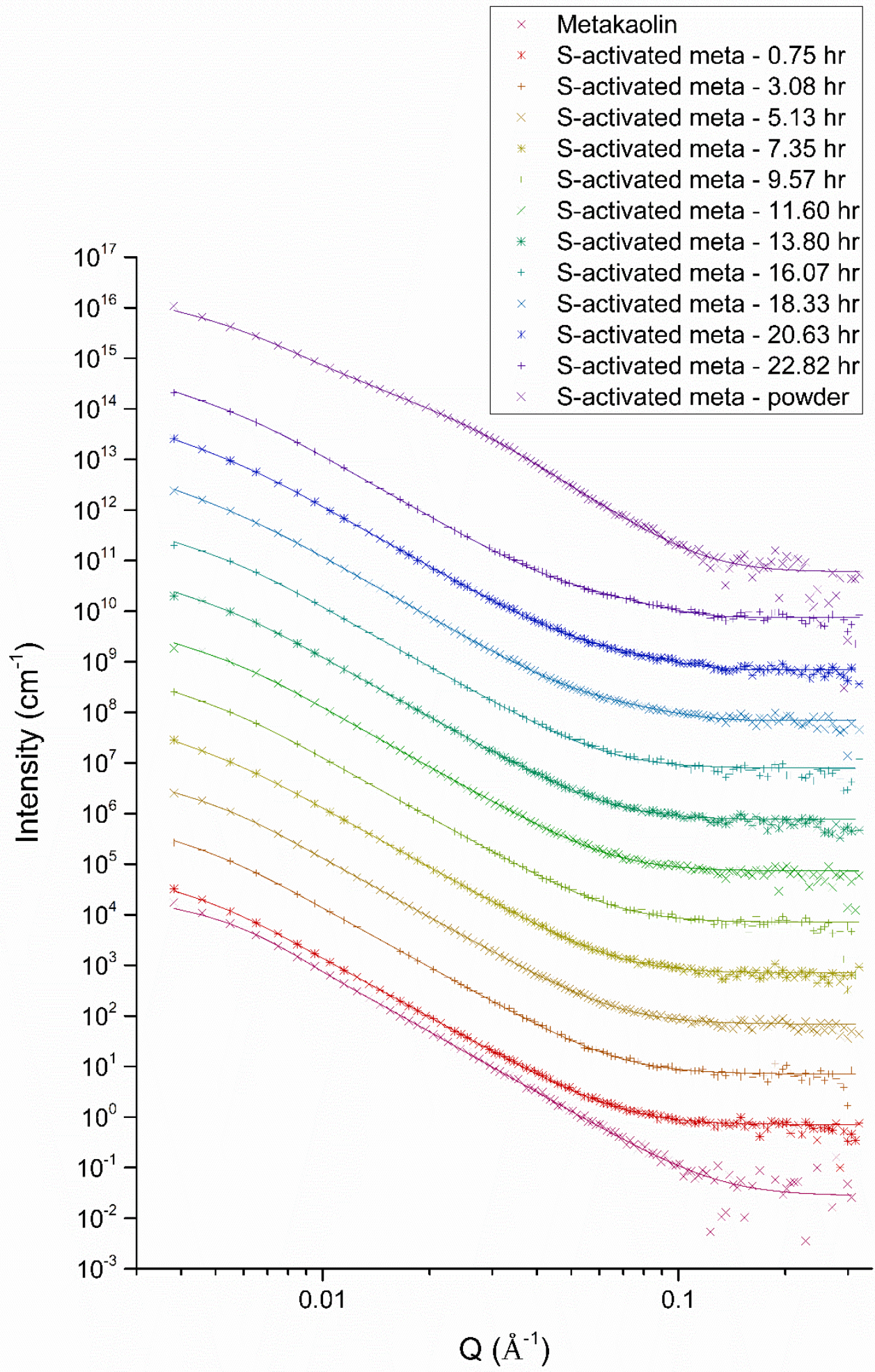

Figure S3. Small-angle neutron scattering pattern of dry metakaolin powder and patterns of the Sactivated metakaolin paste during the alkali-activation reaction. The lines represent the unified fit results. Error bars are excluded for clarity, but are comparable to those given in Figure 9 in the main article. Data are offset by multiples of ten. 


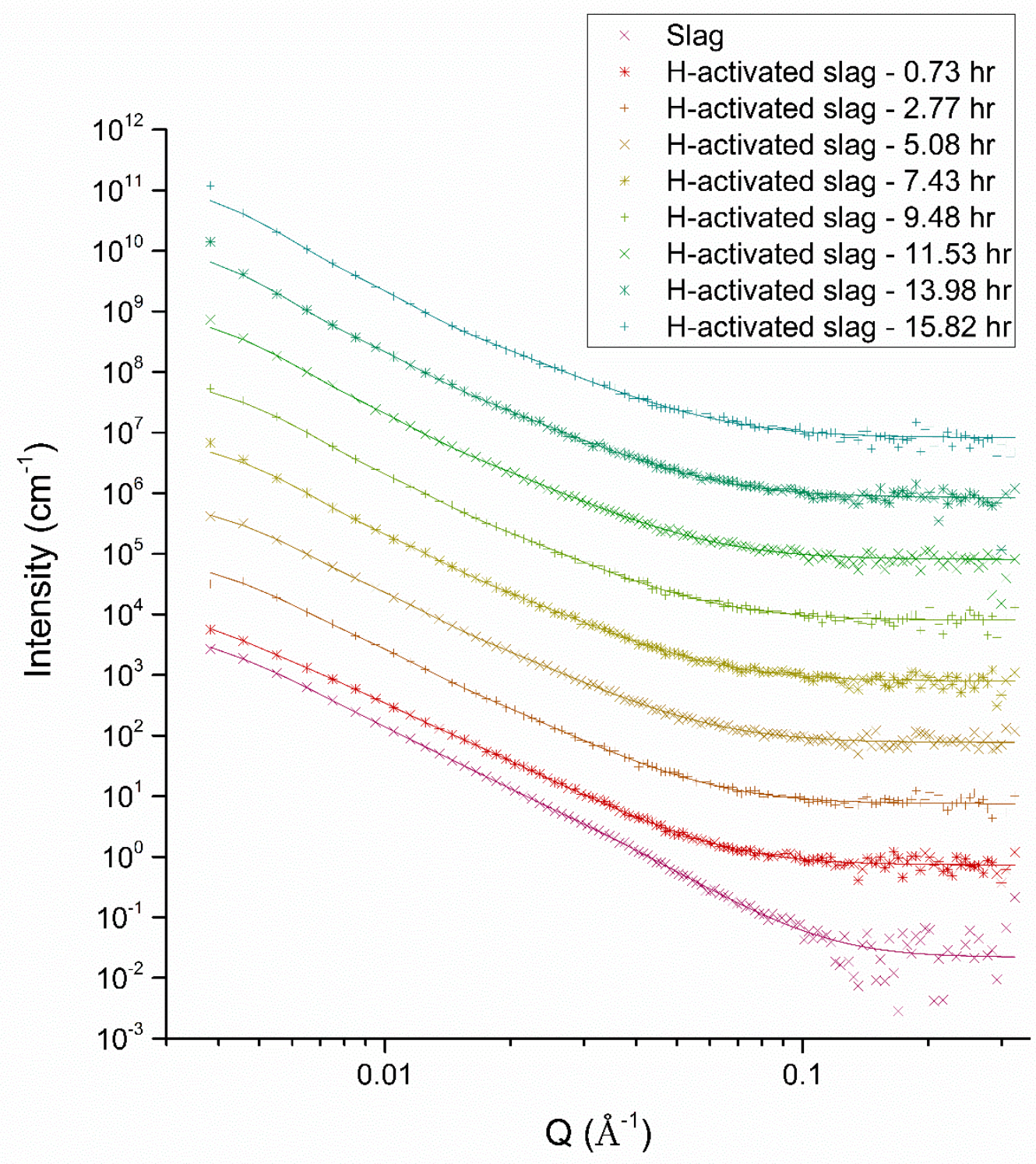

Figure S4. Small-angle neutron scattering pattern of dry slag powder and patterns of the $\mathrm{H}$ activated slag paste during the alkali-activation reaction. The lines represent the unified fit results. Error bars are excluded for clarity, but are comparable to those given in Figure 9 in the main article. Data are offset by multiples of ten. 


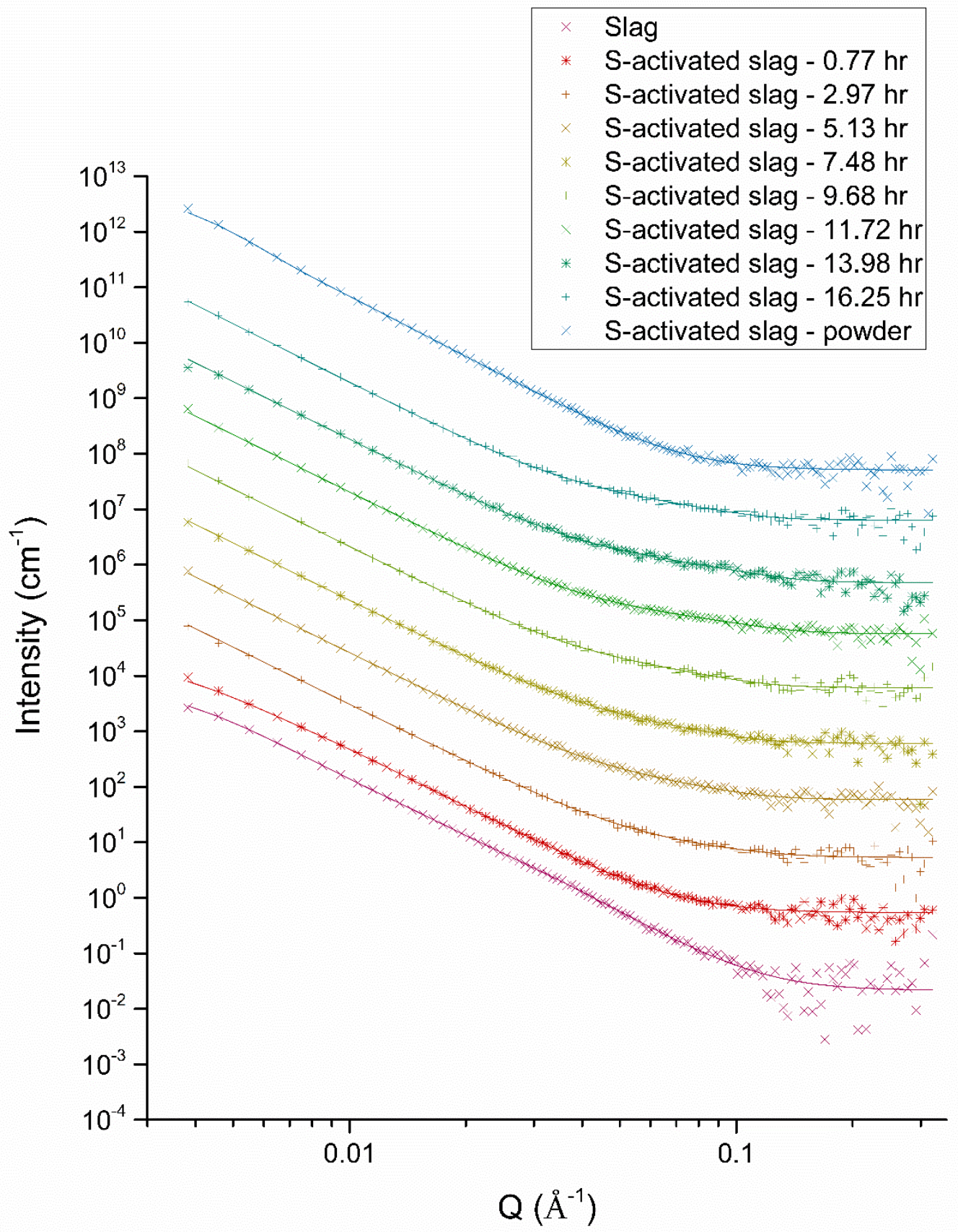

Figure S5. Small-angle neutron scattering pattern of dry slag powder and patterns of the Sactivated slag paste during the alkali-activation reaction. The lines represent the unified fit results. Error bars are excluded for clarity, but are comparable to those given in Figure 9 in the main article. Data are offset by multiples of ten. 


\section{Porod Plots of the Alkali-Activation Reaction}

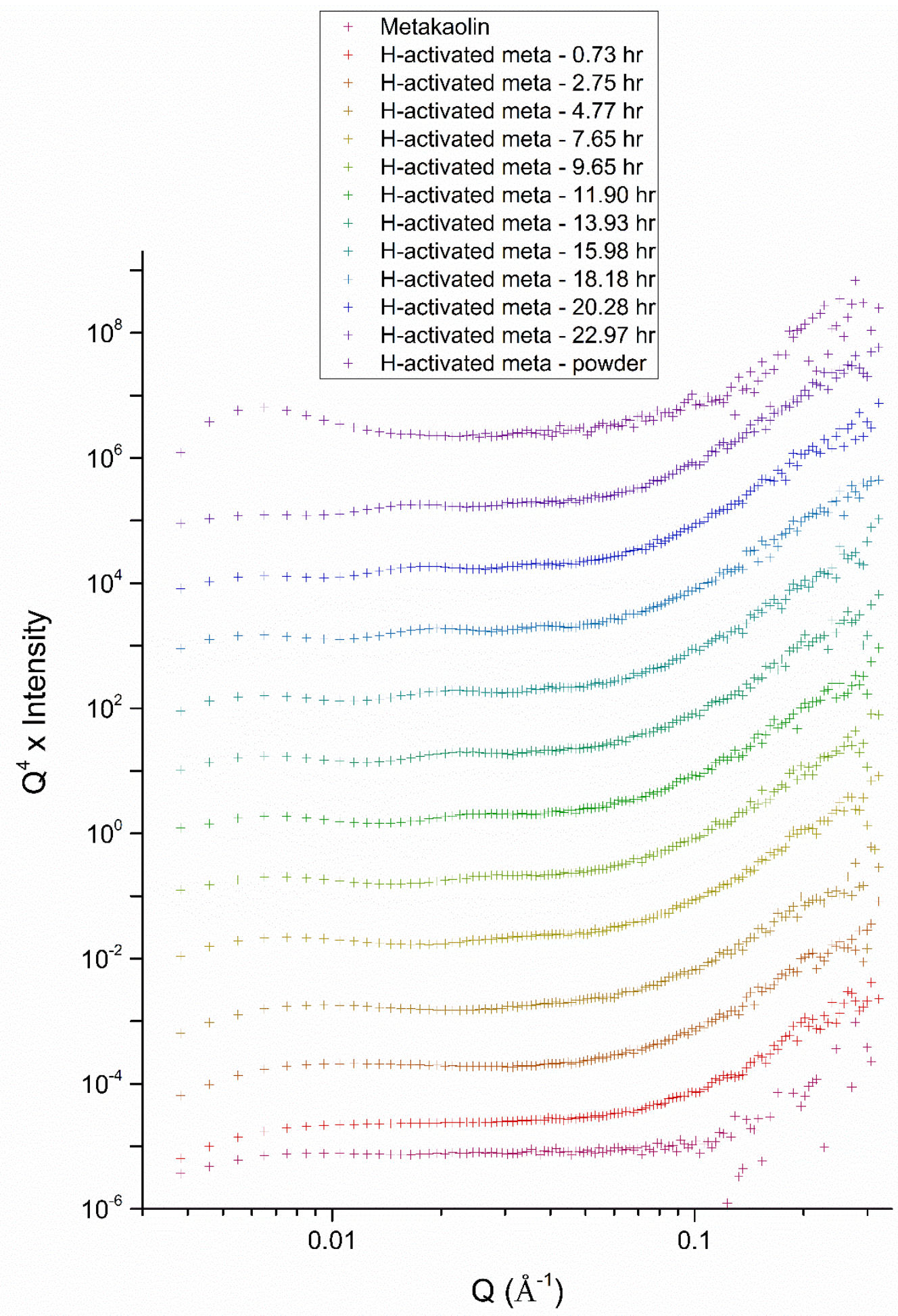

Figure S6. Porod plot of dry metakaolin powder and plots of the H-activated metakaolin paste during the alkali-activation reaction. Data are offset by multiples of ten. 


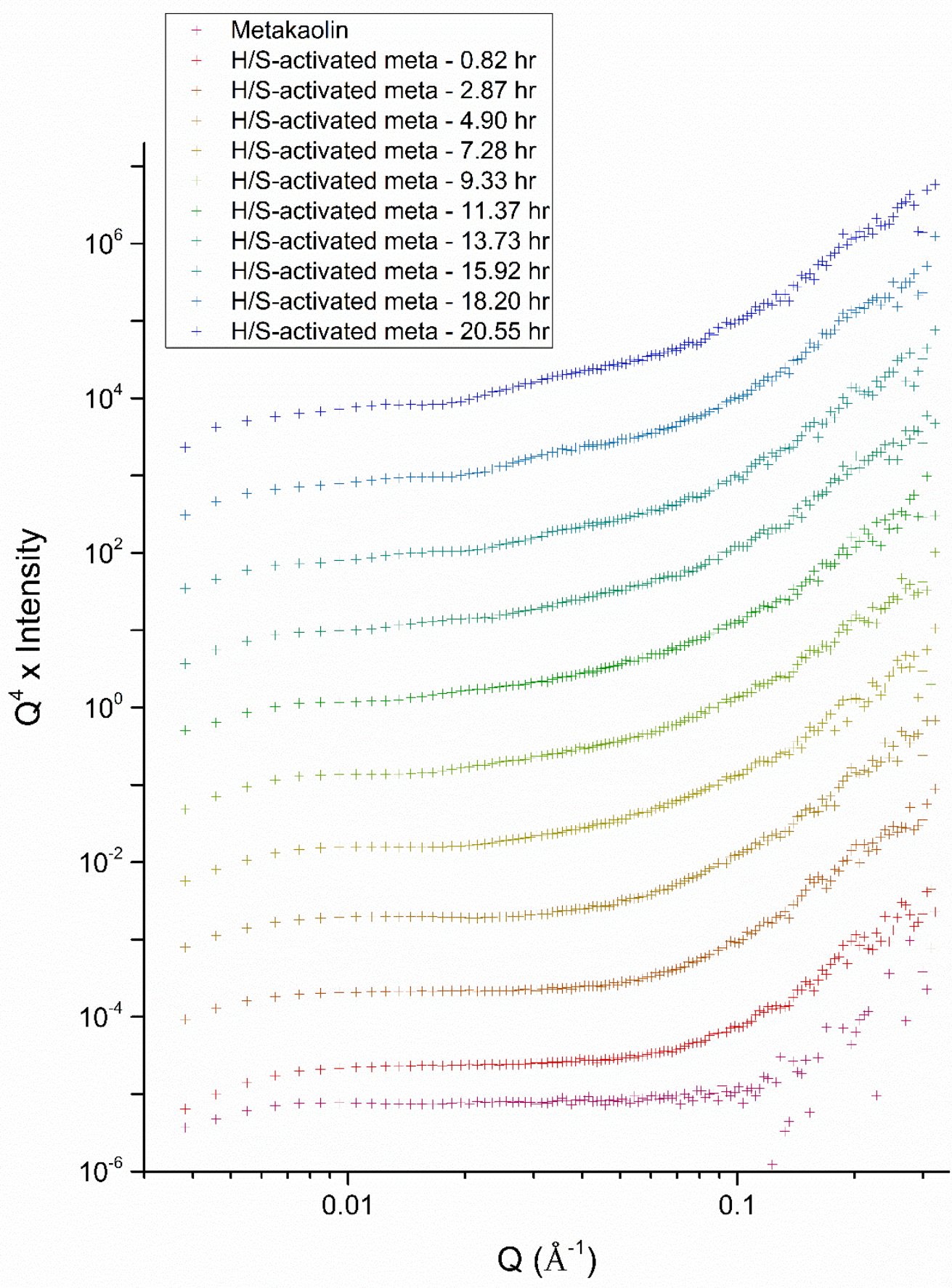

Figure S7. Porod plot of dry metakaolin powder and plots of the H/S-activated metakaolin paste during the alkali-activation reaction. Data are offset by multiples of ten. 


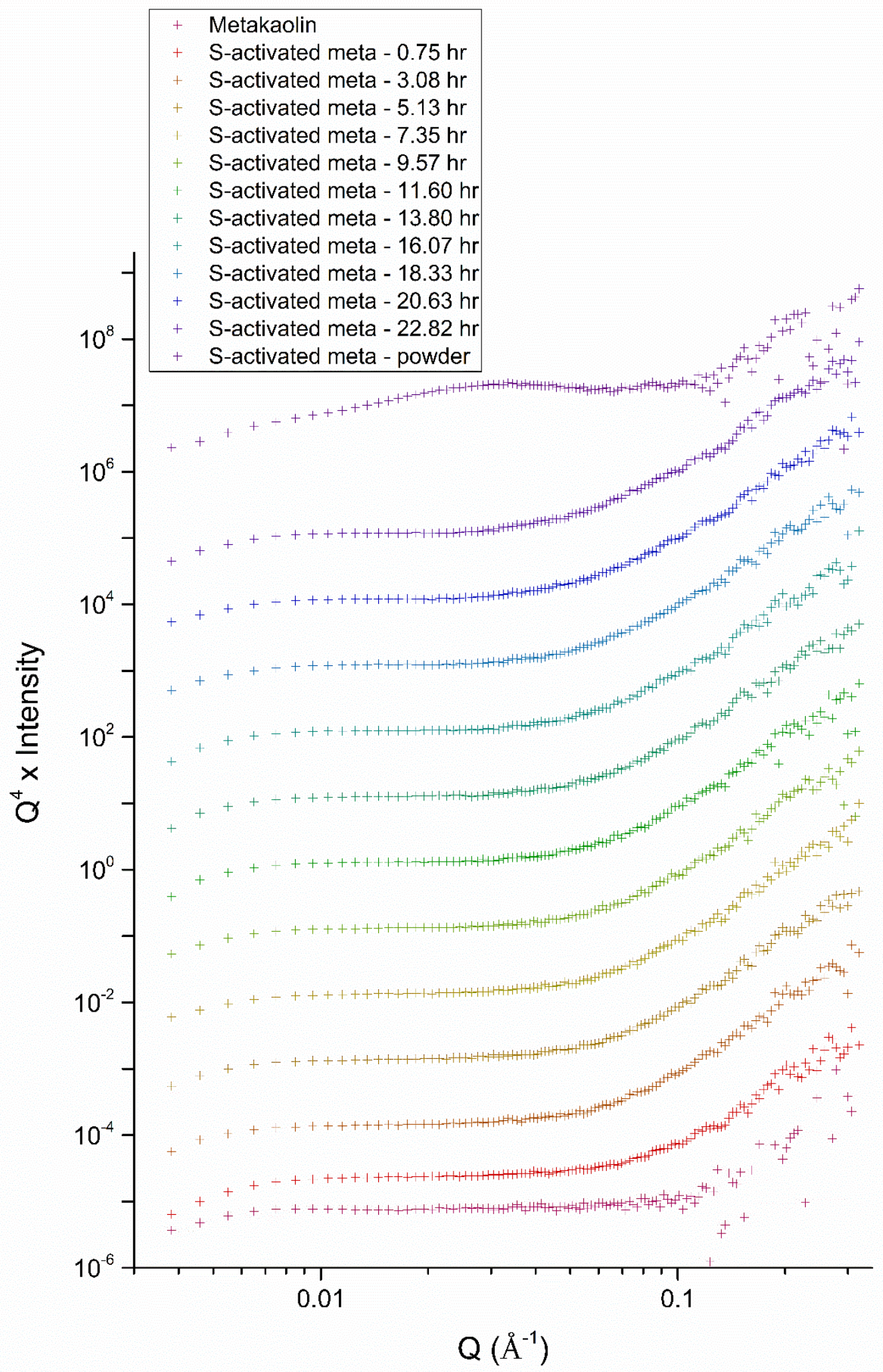

Figure S8. Porod plot of dry metakaolin powder and plots of the S-activated metakaolin paste during the alkali-activation reaction. Data are offset by multiples of ten. 


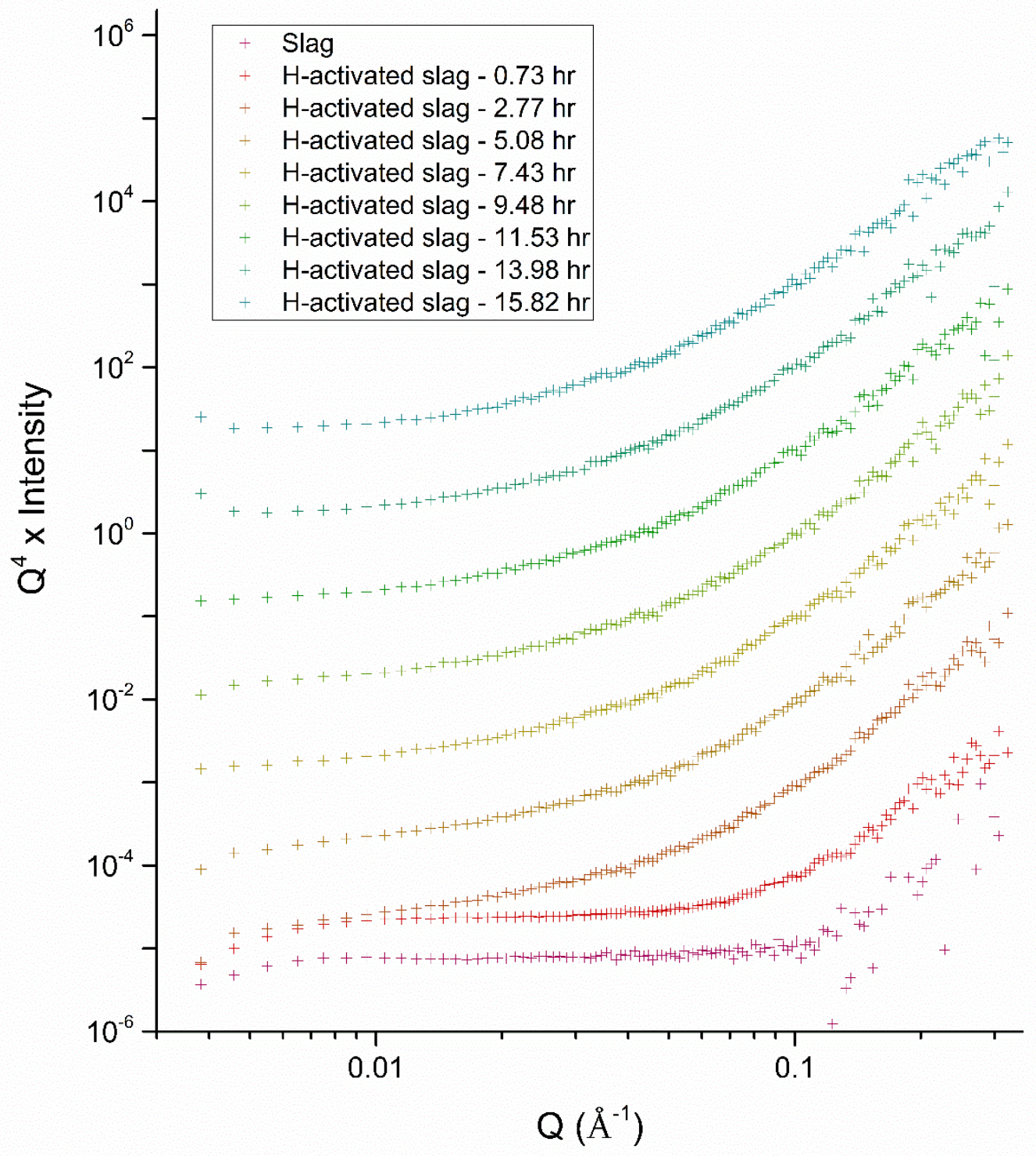

Figure S9. Porod plot of dry slag powder and plots of the H-activated slag paste during the alkali-activation reaction. Data are offset by multiples of ten. 


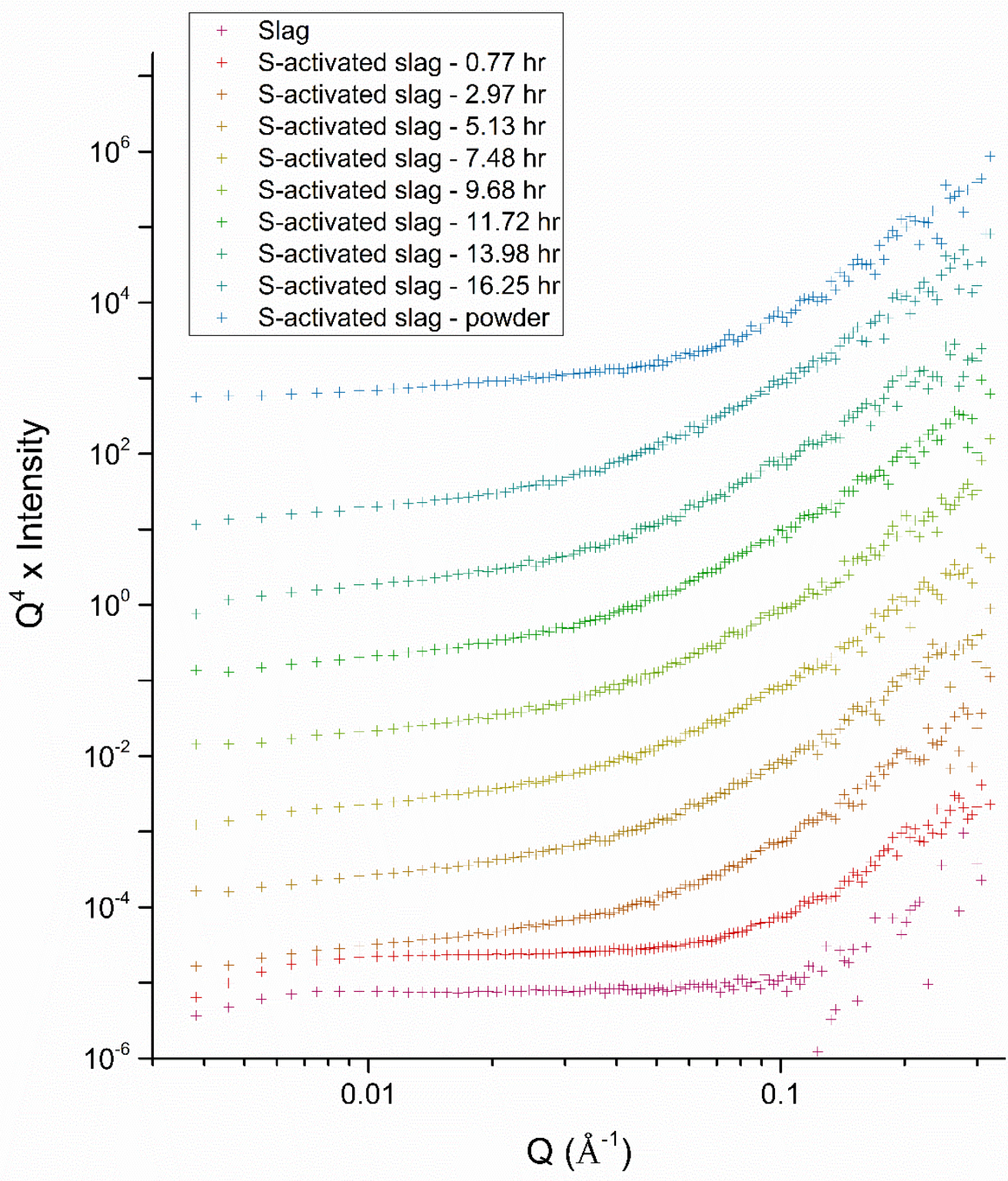

Figure S10. Porod plot of dry slag powder and plots of the S-activated slag paste during the alkali-activation reaction. Data are offset by multiples of ten. 


\section{Kratky Plots of the Alkali-Activation Reaction}

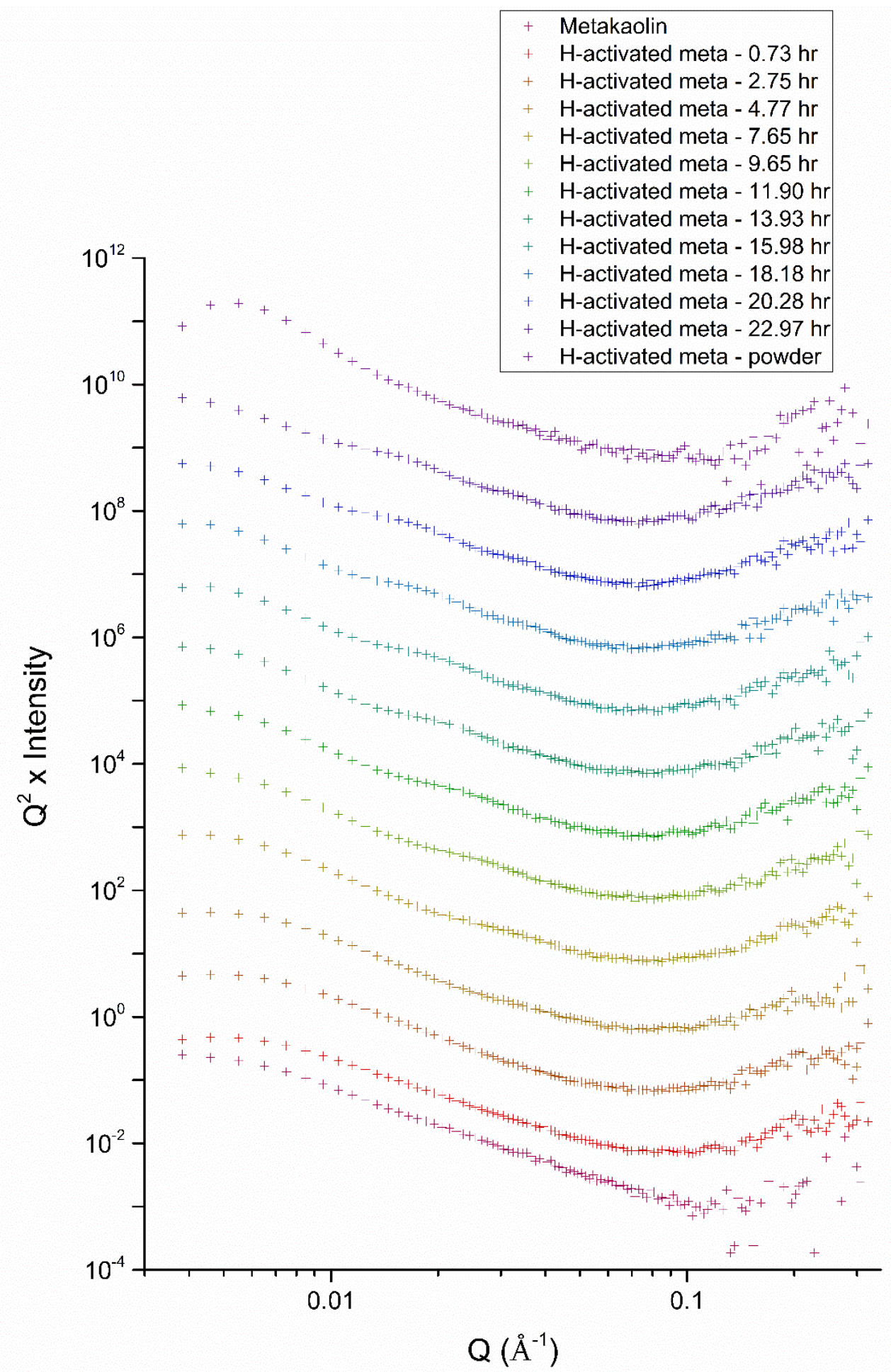

Figure S11. Kratky plot of dry metakaolin powder and plots of the $\mathrm{H}$-activated metakaolin paste during the alkali-activation reaction. Data are offset by multiples of ten. 


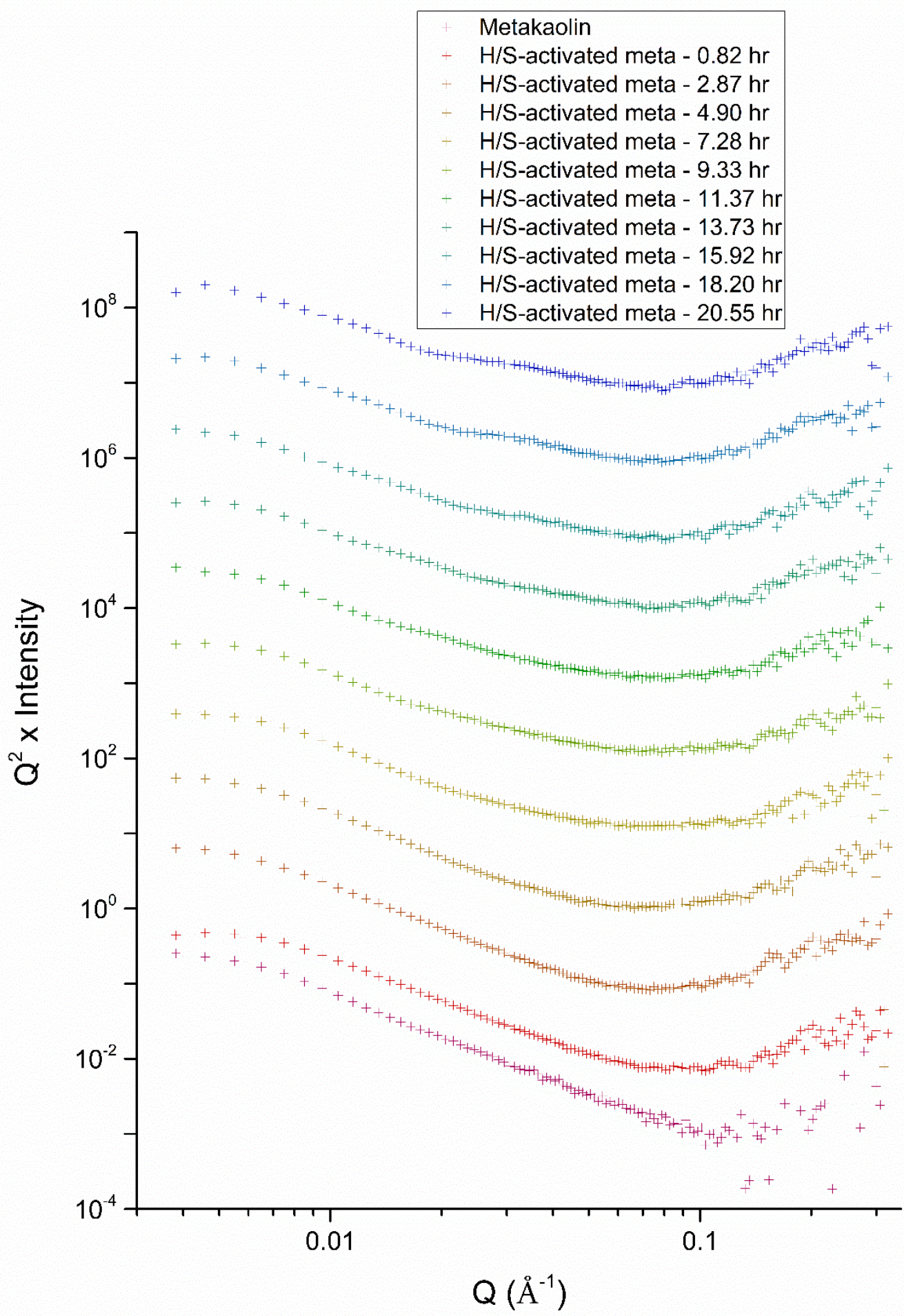

Figure S12. Kratky plot of dry metakaolin powder and plots of the H/S-activated metakaolin paste during the alkali-activation reaction. Data are offset by multiples of ten. 


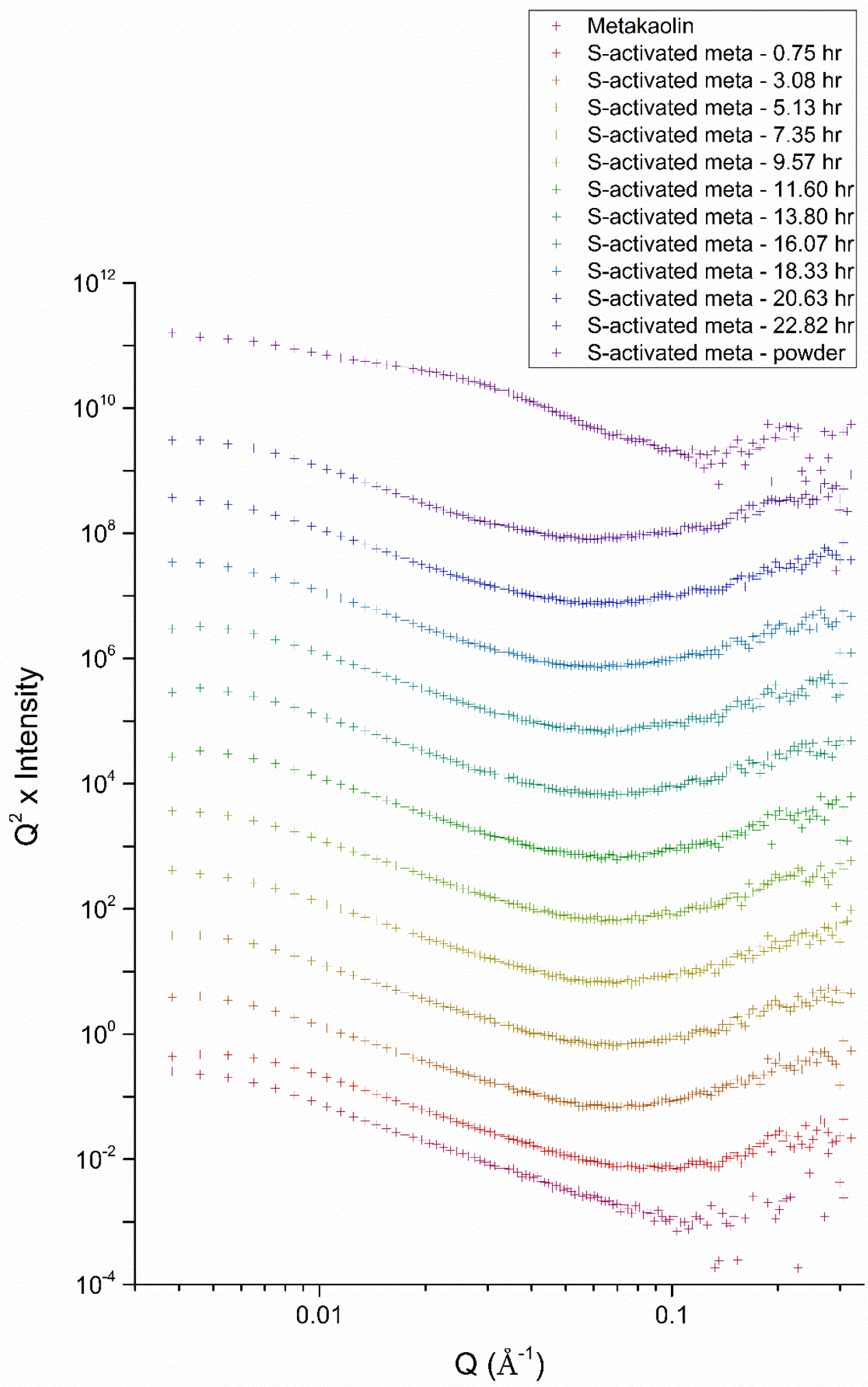

Figure S13. Kratky plot of dry metakaolin powder and plots of the S-activated metakaolin paste during the alkali-activation reaction. Data are offset by multiples of ten. 


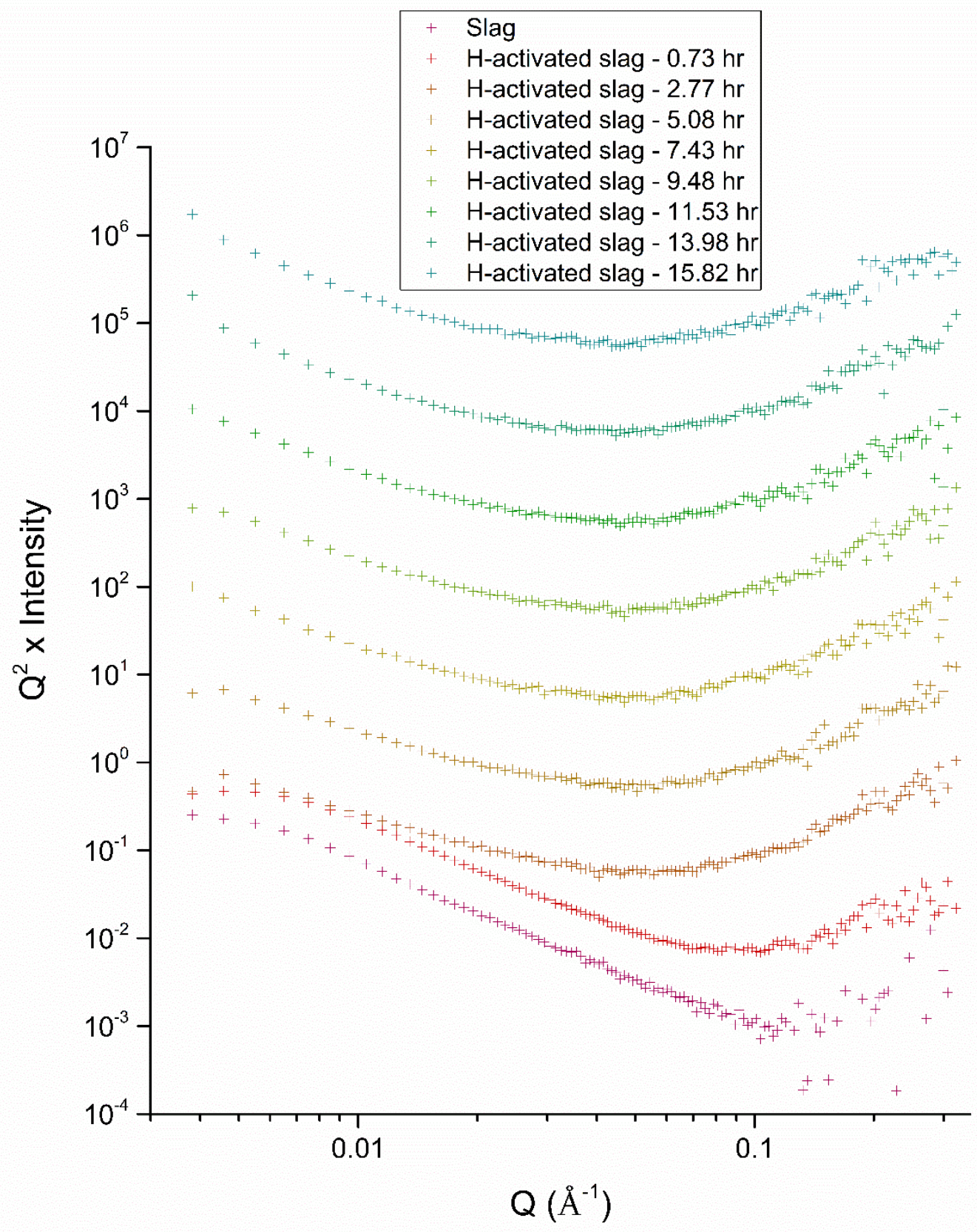

Figure S14. Kratky plot of dry slag powder and plots of the H-activated slag paste during the alkali-activation reaction. Data are offset by multiples of ten. 


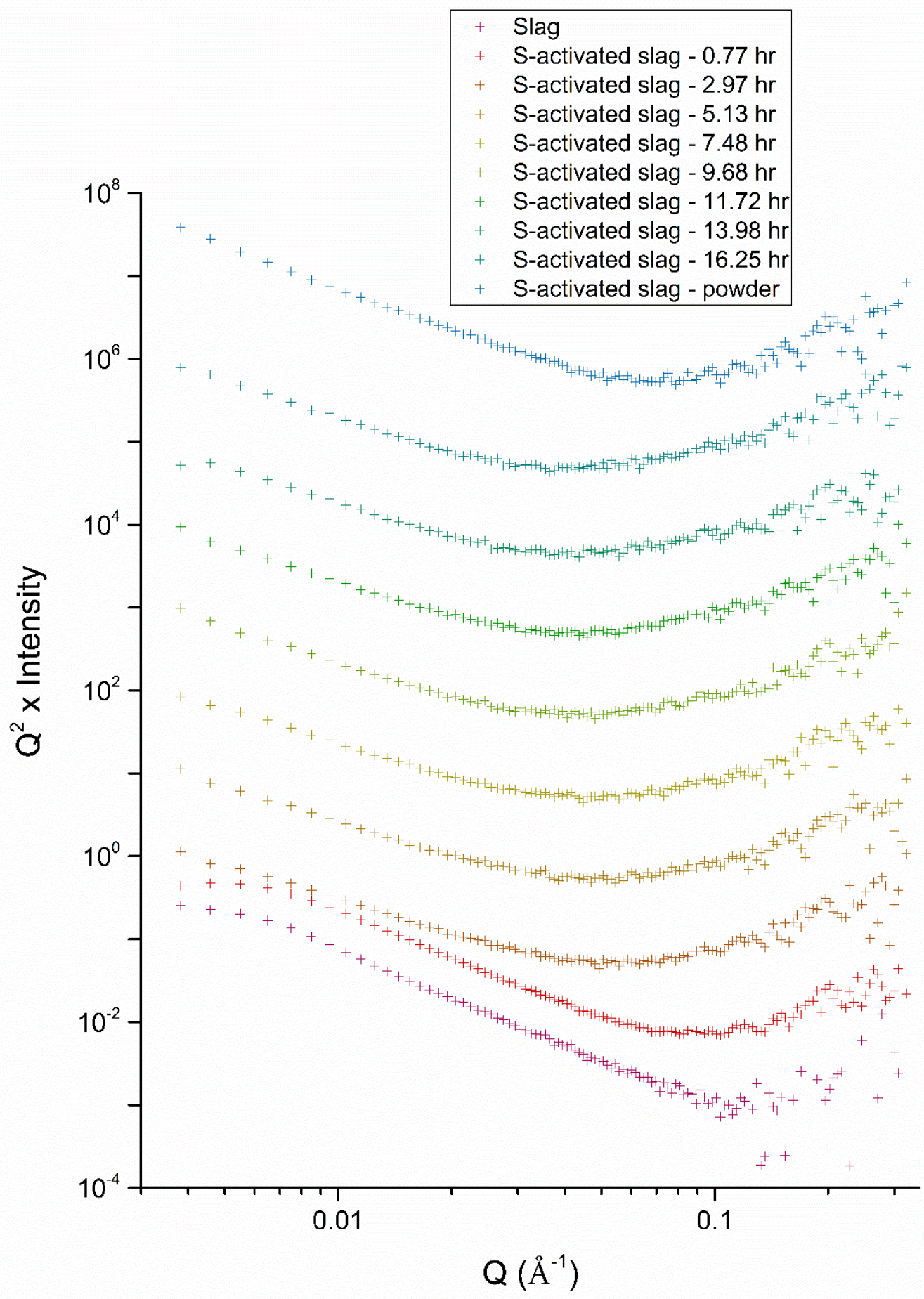

Figure S15. Kratky plot of dry slag powder and plots of the S-activated slag paste during the alkali-activation reaction. Data are offset by multiples of ten. 


\section{S-activated Metakaolin - Powder}

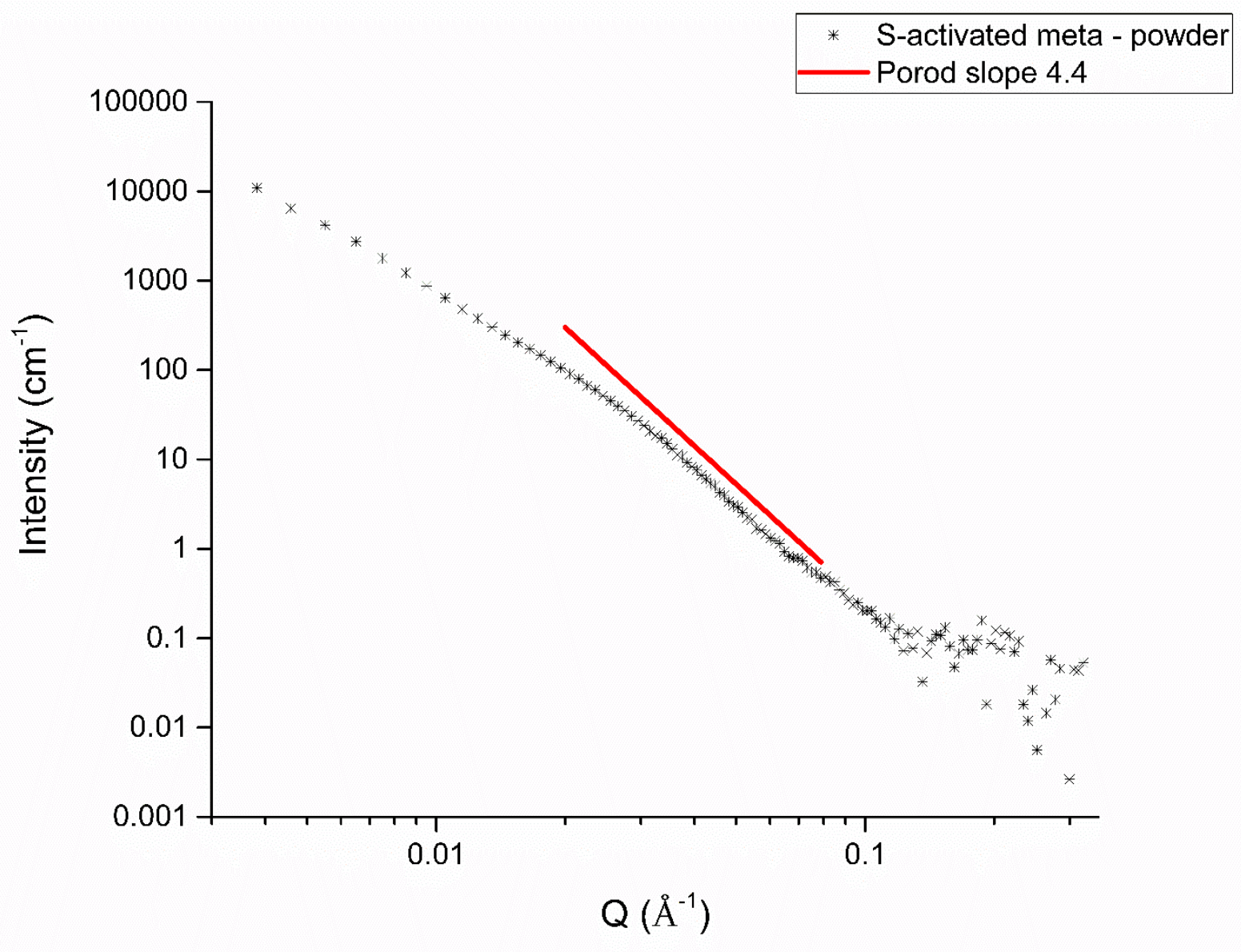

Figure S16. Small-angle neutron scattering pattern of S-activated metakaolin powder (90 days) and a Porod line with a slope of 4.4.

\section{Polydispersity Fitting}

Given that the AAM pastes consist of a range of pore sizes from the nanometer to the micron length scale, the size-distribution solving Irena macro (Ilavsky and Jemian, 2009; Potton et al., 1988) at first seems a natural choice for such a system, as the only inherent assumptions of this model are that the scattering originates from the summation of individual scattering populations (i.e., spheres with a given radius) weighted according to their number density in the sample. In systems where there exist significant 
contributions to the small angle scattering from features with characteristic sizes just above (larger than) the regime directly accessed by the measurement, such that there is no associate Guinier plateau can be seen, the size-distribution macro often fails. One technique to mitigate such long-featured contributions to the data is to subtract from the original data a Porod-like slope, attributed to a larger-than-seen scatterer population, producing a reduced data set with previously subtle features now clearly defined (see Figure S1). Using this technique, we were able to successfully model some, but not all, of the measured datasets, as this approach fails when there is little to no distinct scattering above a power-law slope. Hence, in the main article we have used the unified fit approach to model the data instead of the polydispersity model outlined here.

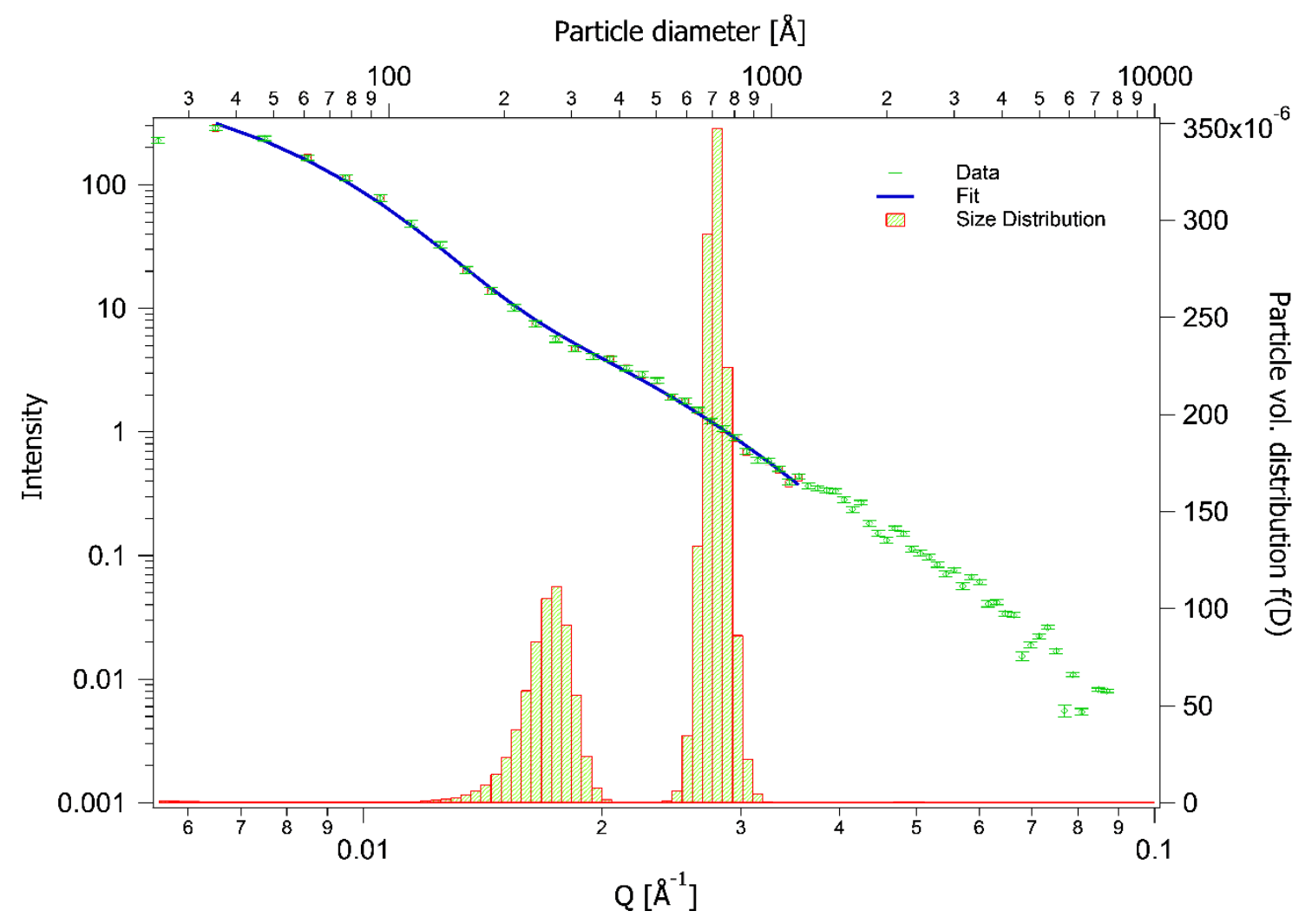

Figure S17. Polydispersity fit to the difference curve obtained by subtracting the $22.97 \mathrm{hr} H$-activated metakaolin SANS data set from the $0.73 \mathrm{hr}$ data set. 


\section{References}

Ilavsky, J. \& Jemian, P. R. (2009). Journal of Applied Crystallography 42, 347-353.

Potton, J. A., Daniell, G. J. \& Rainford, B. D. (1988). Journal of Applied Crystallography 21, 663668. 